\title{
Initial performance assessment of hybrid inflatable structures ${ }^{\text {is }}$
}

\author{
Scott J.I. Walker*, Anthony D. McDonald, Toshihiko Niki, Guglielmo S. Aglietti \\ University of Southampton, Southampton, United Kingdom
}

\section{A R T I C L E I N F O}

\section{Article history:}

Received 12 January 2010

Received in revised form

22 September 2010

Accepted 10 October 2010

Available online 6 November 2010

Keywords:

Inflatable structures

Tape springs

Deployable structures

\begin{abstract}
A B S T R A C T
Inflatable technology for space applications is under continual development and advances in high strength fibers and rigidizable materials have pushed the limitations of these structures. This has lead to their application in deploying large-aperture antennas, reflectors and solar sails. However, many significant advantages can be achieved by combining inflatable structures with structural stiffeners such as tape springs. These advantages include control of the deployment path of the structure while it is inflating (a past weakness of inflatable structure designs), an increased stiffness of the structure once deployed and a reduction in the required inflation volume. Such structures have been previously constructed at the Jet Propulsion Laboratory focusing on large scale booms. However, due to the high efficiency of these designs they are also appealing to small satellite systems.

This article outlines ongoing research work performed at the University of Southampton into the field of small satellite hybrid inflatable structures. Inflatable booms have been constructed and combined with tape spring reinforcements to create simple hybrid structures. These structures have been subjected to bending tests and compared directly to an equivalent inflatable tube without tape spring reinforcement. This enables the stiffness benefits to be determined with respect to the added mass of the tape springs. The paper presents these results, which leads to an initial performance assessment of these structures.
\end{abstract}

(c) 2010 Elsevier Ltd. All rights reserved.

\section{Introduction}

Inflatable technology has been used in aerospace applications over many decades as the ability to deploy large structures with a low mass and very high packing efficiency has always been a requirement in this industry. Flight heritage of these structures began in the 1950s with the Echo Balloon series, which were designed as passive spacebased communications reflectors. With recent advances in high strength fibers and rigidizable materials [1], inflatable technology has now matured into the space age as demonstrated by NASA's large-scale inflatable spacecraft 'TransHab' [2]. However, these structures are not only useful for

\footnotetext{
This paper was presented during the 60th IAC in Daejeon.

* Corresponding author.

E-mail address: sjiw@soton.ac.uk (S.J. Walker).
}

large scale systems but may also be a very elegant solution for small scale deployment applications.

\section{Space inflatable booms}

There are a wide range of potential applications of inflatable structures such as solar arrays, radar and reflector arrays, solar concentrators, solar shades and solar sails and all of these concepts require structural support which generally takes the form of a circular section inflatable boom. Since the 1980s a significant amount of research has been performed studying various configurations of inflatable booms as both standalone components and parts of area deployment devices and antennae [3]. In recent years this work has been led by the Jet Propulsion Laboratory (JPL) in conjunction with two specialist companies, L'Garde and ILC Dover based in the USA [4]. Various specifics of the 
inflatable booms have been investigated to identify the best materials for space applications and potential weaknesses of these structures. It was concluded that they are most structurally efficient when combined with metal stiffeners known as tape springs [3]. Tape springs (defined as thin metallic strips with an initially curved cross section) have already been investigated in the space industry as hinges/hinge components by many companies (Surrey Satellite Technology Limited [5], Metravib [6], etc.). However, the combination of inflatable material and tape springs creates a hybrid structure that has a superior performance when compared to the individual components. The tape springs help to guide the deployment and prevent localised compression when the structure is subjected to a load and the inflatable structure increases the booms second moment of area by distancing the tape springs from the neutral axis. Examples of these structures have been built by JPL that have a diameter of $0.076 \mathrm{~m}$ and a length of $5 \mathrm{~m}$. Further work is underway to develop this technology for larger deployments as required by the American space industry [3]. However, they have not been investigated and optimized for small satellite applications with more limited financial budgets, more common in the UK and European market. More specifically, there is limited data available as to the structural performance improvement of these systems with respect to the added mass penalty. The aim of this publication is to address this by experimentally testing a range of boom configurations and determining the buckling load. This enables the stiffness and mass tradeoff to be initially quantitatively assessed.

\section{Experimental development and material testing}

Modern inflatable structures for aerospace applications are mostly constructed from advanced fibers such as Vectran and Kevlar along with more conventional fibers such as Nylon [3]. These fabrics have been used with coatings such as polyurethane and provide the main structural resistance to the internal inflation pressure. The main air tight seal is provided by an internal bladder made from materials such as polyurethane. Rigidization of the inflated booms can be achieved by using specific fabric coatings or through the use of specialized materials such as aluminium laminate [3]. However, rigidization methods are beyond the scope of this initial research.

Through initial investigations into sourcing these modern fabrics it was found to be very costly to purchase the small amounts of the materials required for this initial research effort. Alternate materials were sourced that are more widely used in terrestrial applications such as sailing. These materials ranged from coated or impregnated Ripstop Nylon and Ripstop Polyester. Although both Nylon and Polyester are fibers that have been used previously in aerospace inflatable applications [3,7], these 'off the shelf' fabrics are not space grade materials. However, the aim of this work is to initially determine the relative stiffness increase of incorporating tape springs into an inflatable boom at a specified pressure. Therefore the use of 'off the shelf fabrics enables the relative stiffnesses to be easily determined for this initial study.
When using fabrics as a structural material it is important to understand the properties of the materials which directly relates back to its method of construction. The synthetic man-made fibers are initially formed into yarn so it can be woven together to make the fabric. The weaving process incorporates longitudinal yarn running along the length of the fabric roll and transverse yarns which are weaved into the longitudinal yarns to create the roll of fabric. The directions of the longitudinal and transverse yarns are known as the warp and fill directions, respectively. Typically the material properties vary between the warp and fill orientations and more significantly in the bias direction which is $45^{\circ}$ in between the warp and fill directions. In this respect these properties are similar to $0^{\circ}$ and $90^{\circ}$ composite laminates.

The initial aim was to test an inflatable tube with a radius of $50 \mathrm{~mm}$, up to an inflation pressure of $103.4 \mathrm{~N} / \mathrm{m}^{2}$ (15 psig). For initial calculation purposes if a material thickness of $0.1 \mathrm{~mm}$ is assumed, an initial estimate into the required hoop stress $\left(\sigma_{H}\right)$ of the material can be calculated. For this geometry, at this pressure the selected material must be able to withstand a hoop stress of 51.7 MPa. Along with the strength requirement the fabrics also need to have the ability of maintaining the required shape when inflated (i.e. avoid 'ballooning').

A range of more than 20 fabrics were obtained and tested in Instron machines using two test samples per fabric to determine the tensile strength and Young's modulus of the samples in the warp direction. This allowed an initial material selection based on ultimate tensile strength, mass and availability before more in-depth testing was performed. Initial inflation tests of small tubes were also performed to identify the materials that were susceptible to ballooning, allowing the most suitable fabric to be selected. This resulted in the selection of 'Fibermax 94'. Fibermax is an advanced sailcloth fabric that is constructed with the maximum number of nylon fibers which is then dipped through a solution of dissolved resin. The resin is cured at a high temperature resulting in very small resin particles being embedded in the weave, restricting its stretch [8]. From the material tests it proved to be one of the optimum 'off the shelf' fabrics in terms of the strength to mass ratio.

This selected material was subsequently tested repeatedly in the Instron machines in both the warp and fill directions to more accurately determine the material properties. Five further samples were tested in the warp direction and five samples were tested in the fill direction. Each sample was cut into $50 \times 200 \mathrm{~mm}$ strips which when clamped resulted in a test length of $100 \mathrm{~mm}$. The samples were extended at a rate of $5 \mathrm{~mm}$ per minute in an Instron machine until destruction as shown in Fig. 1. The results for all seven tests in the warp direction are displayed in Fig. 2 and the five fill direction test results are shown in Fig. 3.

It can be seen from Fig. 2 that the stress-strain relationship for the warp direction is non-linear, which is common for fabrics due to the weave construction [9]. Various gradients could be used to determine a value of Young's modulus, the simplest being the gradient between the zero stress point and the point of failure. For the data shown in Fig. 2 this simple approach to determine Young's modulus 


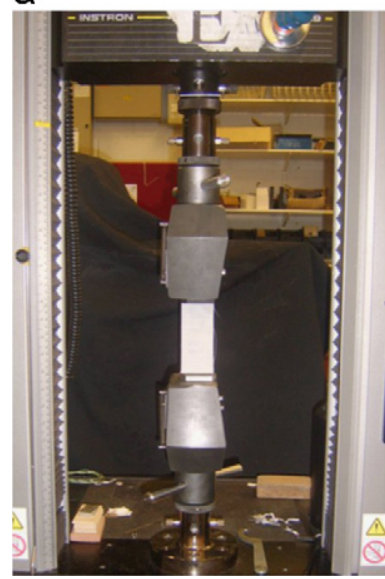

b

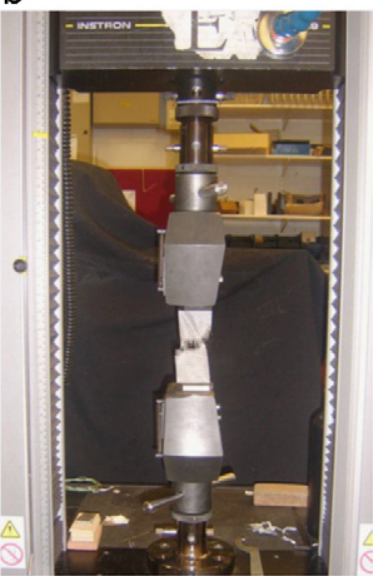

Fig. 1. Fabric material testing using an Instron machine.

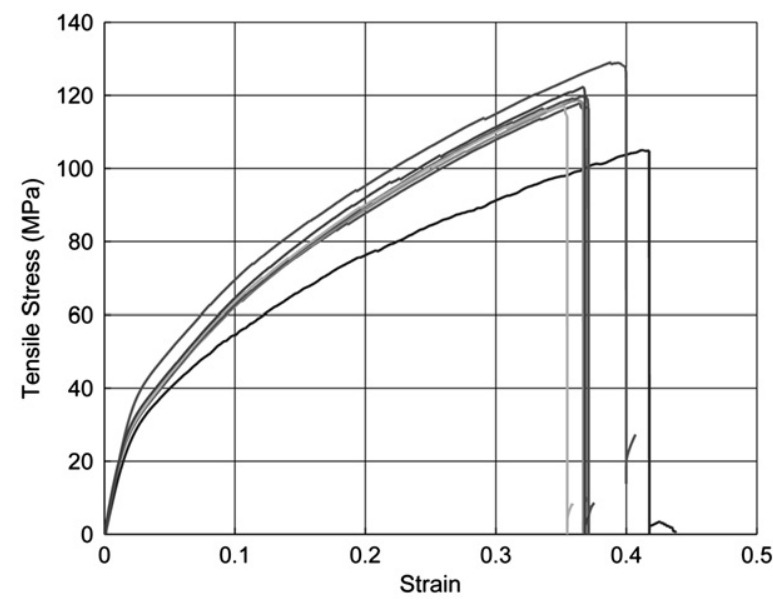

Fig. 2. Stress/strain properties for Fibermax 94 in the warp direction.

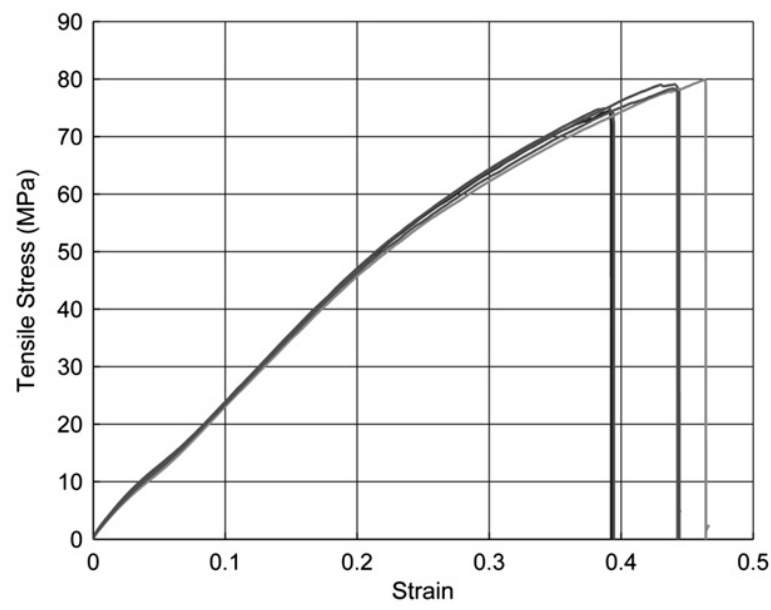

Fig. 3. Stress/strain properties for Fibermax 94 in the fill direction.

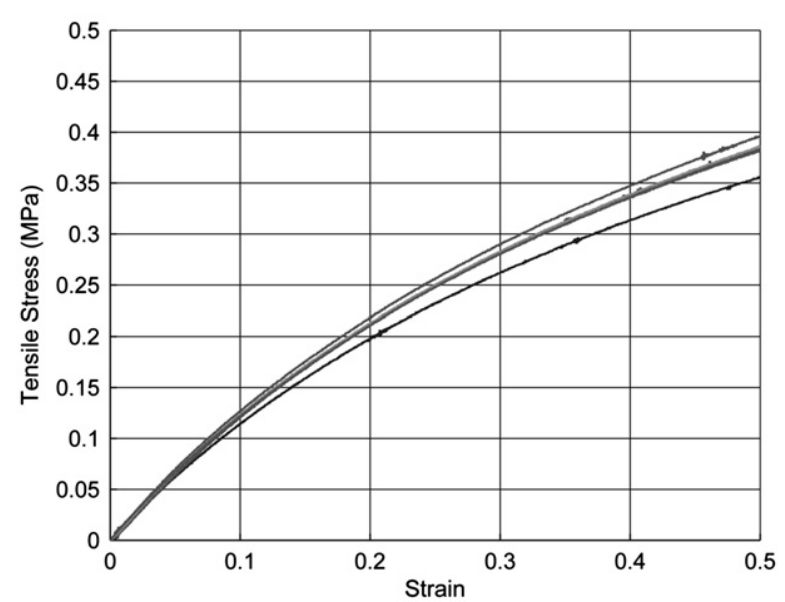

Fig. 4. Stress/strain properties for latex rubber.

produces values ranging from 0.22 to $0.29 \mathrm{GPa}$, with a mean average value of $0.28 \mathrm{GPa}$. The equivalent average Young's modulus for the fill direction is $0.188 \mathrm{GPa}$. The average ultimate tensile stresses for the warp and fill directions are 119 and $77 \mathrm{MPa}$, respectively.

To identify the potential variability in the gradient the warp direction curve can be analysed over different strain regions giving a complete gradient range from 0.125 to 1.6 GPa. If the anticipated stress/strain is low then a higher Young's modulus value should be used. For higher levels of strain a function should ideally be used to model this relationship. However, permanent deformation strain in the material would alter this function, so careful material testing is required. These complexities are beyond the scope of this initial study but are currently under investigation.

The magnitude of stress/strain experienced by the fabric is dependant on the material used for the bladder. The choice of material for the inner bladder was limited by the properties required of it, i.e. being non-permeable and able to expand to the internal shape of the tube. An ideal material that met these requirements and that was freely available was latex rubber. Two thicknesses were initially tested and the lower thickness of $0.5 \mathrm{~mm}$ was finally selected due to its expansion properties. Five samples were tested in the Instron machine using the same sample size as previously described. The results are shown in Fig. 4.

It can be seen that the rubber easily expands allowing the majority of the inflation stresses to be taken by the outer fabric as required.

\section{Experimental testing}

In order to investigate the deflection of hybrid structures, inflatable tubes were constructed with a radius of $50 \mathrm{~mm}$. This dimension allows easy access to internal seams but also minimises the size of the supporting structure. The fabric tubes were constructed from two fabric areas, one creating the circular end and the other the longitudinal tube. These were sewn together using a simple 
straight stitch with one seam running along the length of the tube. The inner bladder was constructed using the same material pattern. However the seams were bonded using a water-based adhesive called Copydex.

The test campaign focused on determining the tip deflection of a cantilevered inflatable tube subjected to a tip load as shown in Fig. 5.

Three test variables were analysed, namely: tube length, inflation pressure and section configuration. Three tube lengths of $0.6,0.4$ and $0.2 \mathrm{~m}$ were each tested at two inflation pressures of 68.95 and $103.4 \mathrm{kN} \mathrm{m}^{-2}$ (10 and 15 psig, respectively). Four section configurations were tested to identify the stiffening effect of various permutations of tape springs. These configurations were:

- an inflatable tube with no tape spring stiffeners

- an inflatable tube with one pair of small tape springs;

- an inflatable tube with one pair of large tape springs;

- an inflatable tube with two large tape spring pairs.

The section configurations are shown graphically in Fig. 6 along with the direction of the applied load $(F)$.

The three parameters that define the section geometry of a tape spring are: radius of curvature, $R$, angle of embrace, $\alpha$ and thickness, $t$. These properties are shown graphically in Fig. 7 and are listed in Table 1 for the tape springs tested. The tape springs are made of steel with the standard material properties.

The hybrid tubes were mounted at the root end to form a cantilever beam (as shown previously in Fig. 5). The root mounting was achieved with two base plates, one inside and one outside the material layers which when screwed together created the air tight seal. This can be seen in Figs. 8 and 9.

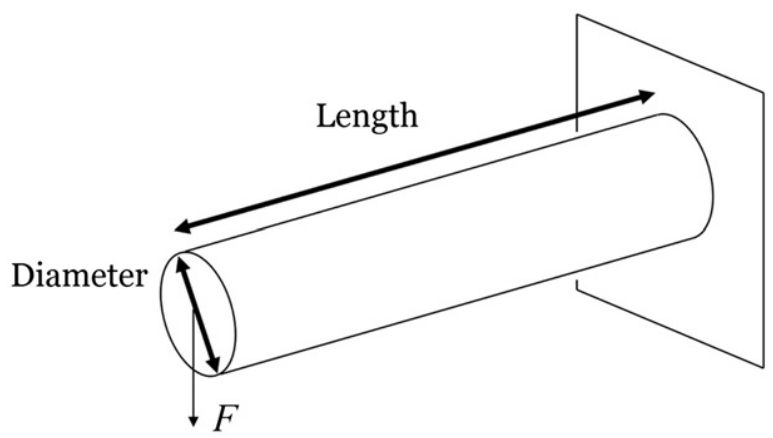

Fig. 5. Schematic of the test configuration.

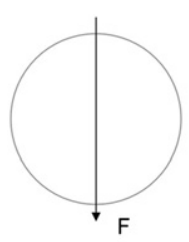

Inf Tube

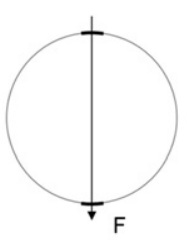

Small Tape Pair

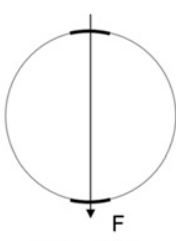

Large Tape Pair

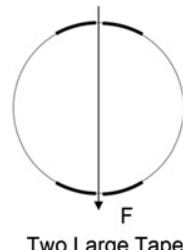

Pairs
Fig. 6. Section configurations shown with applied load, F.

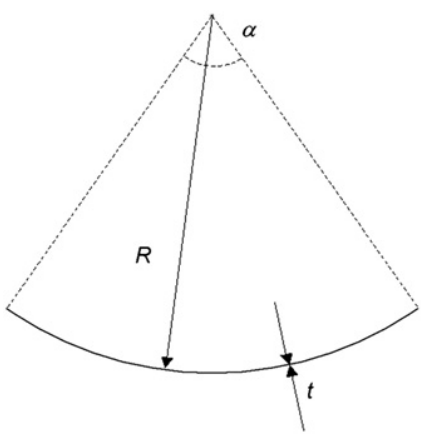

Fig. 7. Cross sectional tape spring parameters.

Table 1

Parameters of tape spring sections.

\begin{tabular}{lcc}
\hline Section parameter & Small tape spring & Large tape spring \\
\hline$R(\mathrm{~mm})$ & 14.7 & 14.5 \\
$\alpha(\mathrm{rad})$ & 0.912 & 1.83 \\
$t(\mathrm{~mm})$ & 0.145 & 0.162 \\
\hline
\end{tabular}

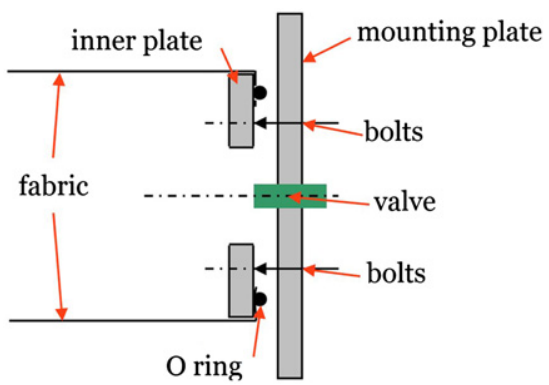

Fig. 8. Schematic of the root mounting.
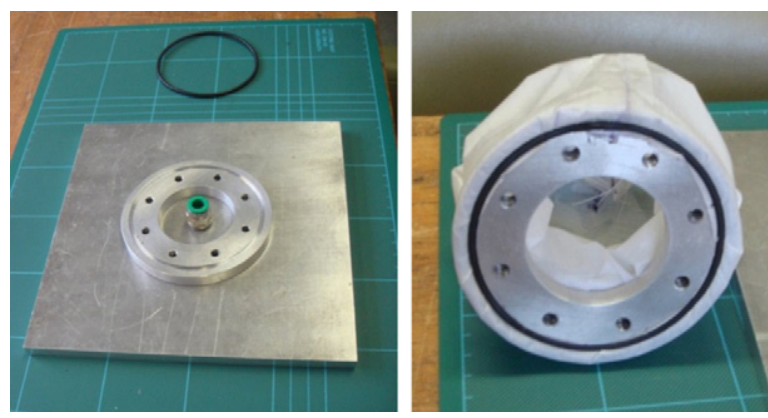

Fig. 9. Photographs of the mounting and inner plates.

A further thin metal plate (with a mass of $46 \mathrm{~g}$ ) was inserted at the tip between the fabric layer and the bladder to spread the applied tip load across the section. This also formed a solid reference point for deflection measurements. The tip loads were applied using suspended weights mounted at the centre of the fabric end area. Deflection measurements were taken with a height gauge which could measure displacements up to $300 \mathrm{~mm}$ with an 


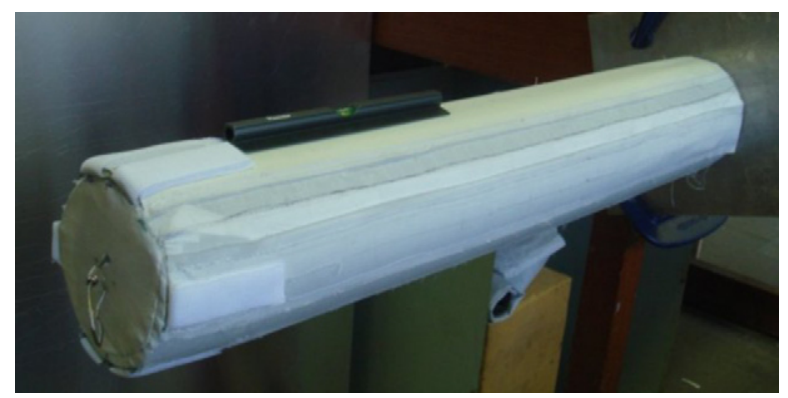

Fig. 10. Experimental configuration shown with spirit level.

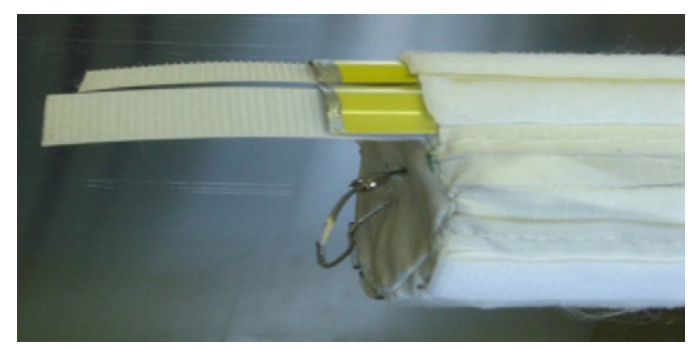

Fig. 11. The open Velcro flaps.

accuracy of $0.01 \mathrm{~mm}$. This experimental configuration is shown in Fig. 10.

As many different section configurations were to be tested, the fabric tubes were designed with sewn pockets running along the length of the tube. These pockets consisted of a second fabric layer sewn on to the main fabric tube of the appropriate width for either the small or large tape springs, allowing the tape springs to be inserted and removed as required. The longitudinal movement of the tape springs relative to the fabric was prevented by using Velcro flaps at the end of the pockets. These Velcro flaps can be seen open in Fig. 11.

Eight pockets were arranged around the circumference as displayed in Fig. 12, four for each tape spring size. The tube was rotated and remounted to achieve the required loading cases as shown previously in Fig. 6. Five repeat deflection tests were performed for each test permutation resulting in a total number of 120 deflection tests.

As there are eight fabric pockets, covering most of the circumference, the majority of the longitudinal tube was constructed of two fabric layers. This creates a more complex stress pattern in the fabric but significantly enhances the functionality of the test specimens which is ultimately desirable for these initial tests. The tape spring root ends were supported using the outer circumference of the inner root plate (this inner root plate is overlaid in Fig. 13), which after buckling resulted in permanent deformation of the tape spring steel.

The tape springs therefore required replacement after every deflection test to failure. Although the tape spring root attachment was not ideal, it was acceptable for the initial tests due to the large number of test permutations required. It should be noted that although various complications in the configuration exist, such as double fabric layers and the root mounting of the tape springs, these issues were deemed acceptable for this first test campaign due to the

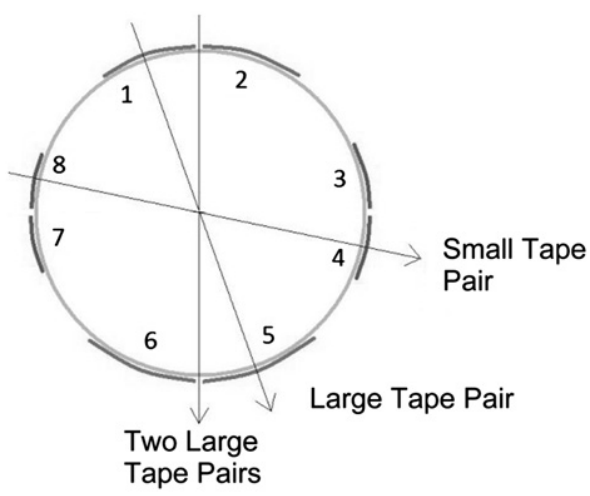

Fig. 12. Tape spring mounting/pocket locations and loading directions.

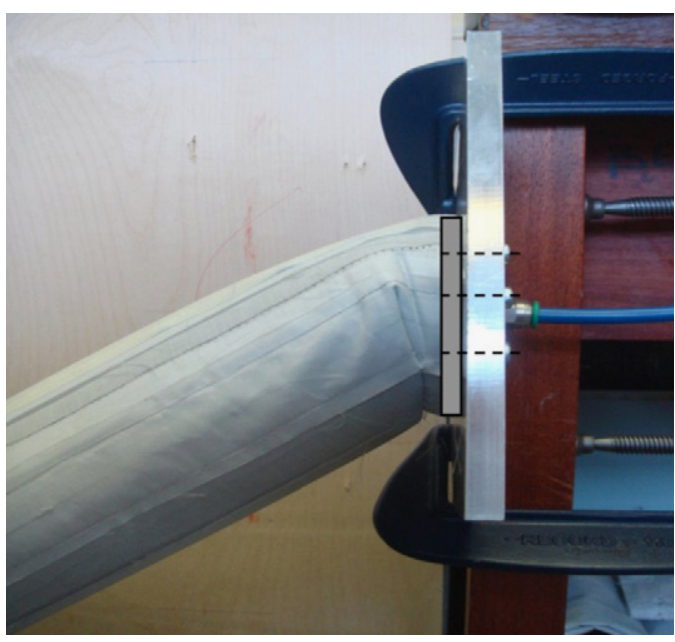

Fig. 13. Inner root plate overlayed on a buckled specimen.

Table 2

Configuration masses (including tip mounted plate).

\begin{tabular}{llll}
\hline Configuration & \multicolumn{2}{l}{ Mass $(\mathrm{g})$ for length } \\
\cline { 2 - 4 } & $0.6 \mathrm{~m}$ & $0.4 \mathrm{~m}$ & $0.2 \mathrm{~m}$ \\
\hline Inf tube & 210 & 163 & 115 \\
Small tape pair & 222 & 171 & 119.2 \\
Large tape pair & 236 & 179.6 & 123.4 \\
Two large tape pairs & 262 & 196.2 & 131.8 \\
\hline
\end{tabular}

significantly improved functionality of the test specimens, and the large number of required test permutations.

At the start of each displacement test the tube was mounted horizontally, checked with a spirit level and the initial tip height was noted. Initially the hook supporting the weights (of mass $0.5 \mathrm{~kg}$ ) was attached and the tip displacement recorded before each weight was added in increments of $1 \mathrm{~kg}$. The tip displacement was recorded after each force increment up to either an excessive displacement after buckling, or the total added mass of $12.5 \mathrm{~kg}$ being reached. The mass of each configuration is 
shown in Table 2, which includes the fabric and bladder layers and the tip mounted metal plate.

\section{Experimental results}

Due to the large quantity of deflection tests performed it is first necessary to determine if the average deflections (over the five repeat tests for each configuration) are representative of the data. The average deflection curves could then subsequently be used for data comparisons. All the individual deflection test results are displayed in Figs. 14-16 along with the averaged result for each tube length and pressure. Although these graphs are densely populated they are only used at this stage to show the applicability of the average result and to identify basic trends.

The deflection data for the $0.2 \mathrm{~m}$ tube is shown in Fig. 14 . From the deflection data for an inflation pressure of $68.95 \mathrm{kN} \mathrm{m}^{-2}$ (10 PSI), Fig. 14(a), the first noticeable trend is the linearity of the data, displaying a clear stiffness relationship between applied load and deflection. It can also be seen that although there is variability in the data, the averages are a good representation of the trends. Some inaccurate individual data points can be identified, but these do not heavily influence the average result due to the number of repeat tests performed.

The effect of the tape spring stiffeners is clearly displayed in this figure. The structure becomes significantly more able to resist buckling. Similar trends can be seen for the $0.2 \mathrm{~m}$ tube at the higher pressure of $103.4 \mathrm{kN} \mathrm{m}^{-2}$ (15 PSI), Fig. 14(b). However for this case the stiffness gradient has increased due to the higher pressure, and the

\section{a}

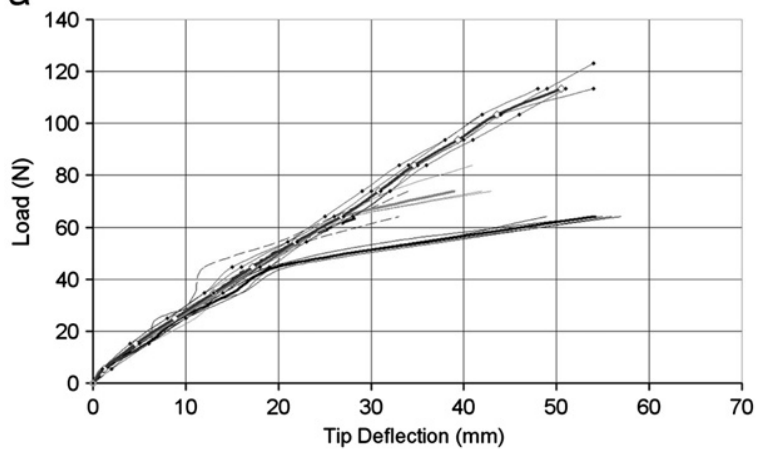

b

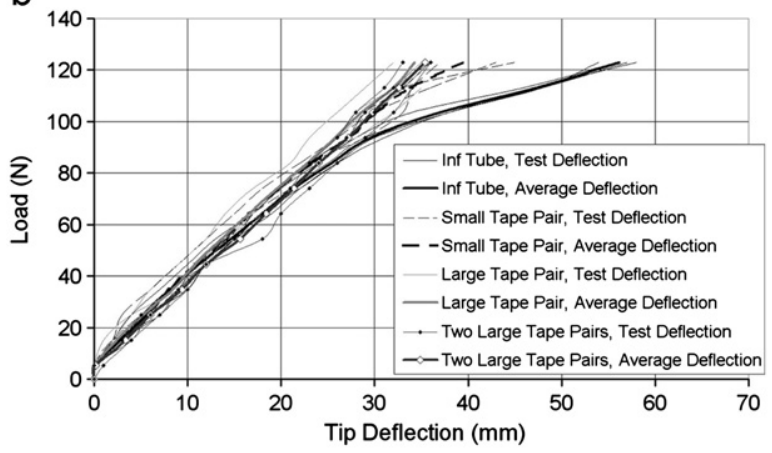

Fig. 14. Tip deflection displacement for $20 \mathrm{~cm}$ tube. a

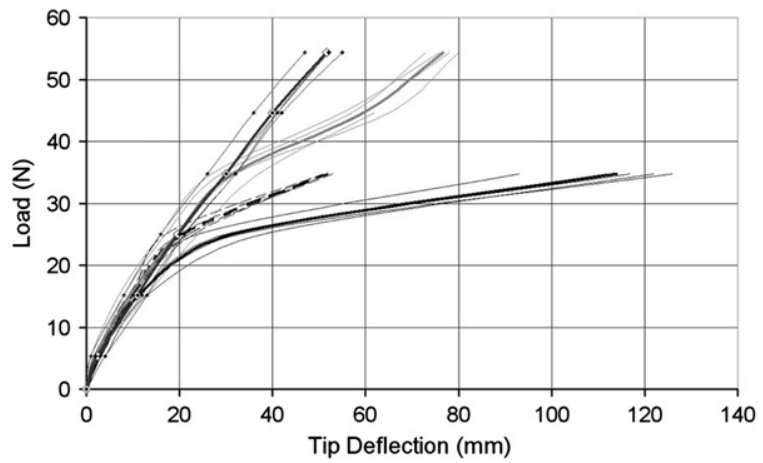

b

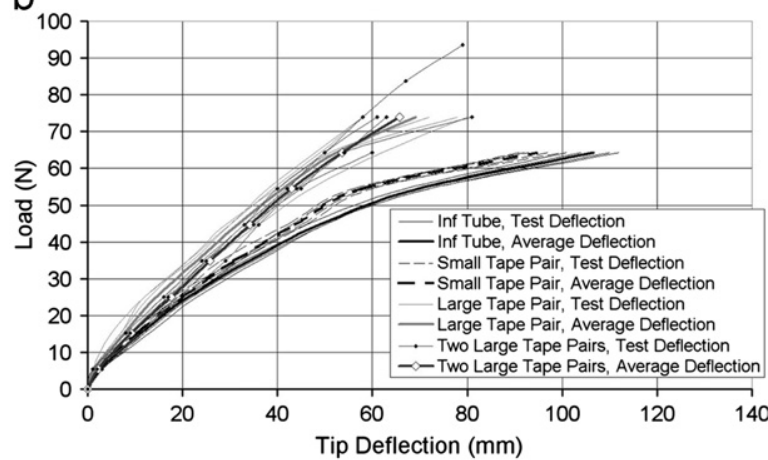

Fig. 15. Tip deflection displacement for $40 \mathrm{~cm}$ tube.

a

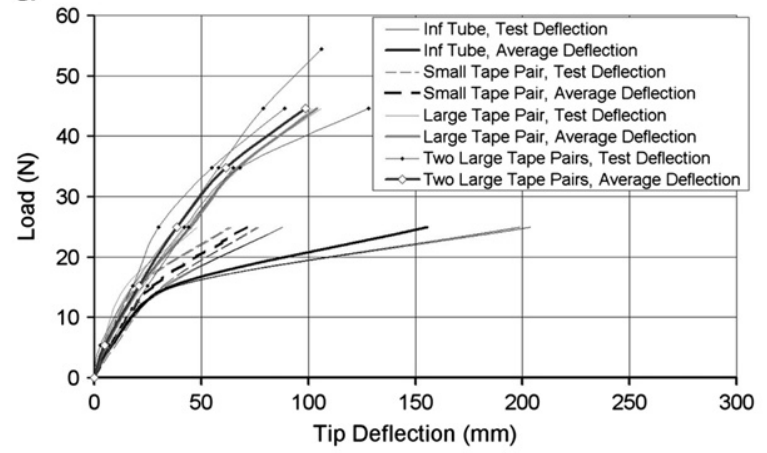

b

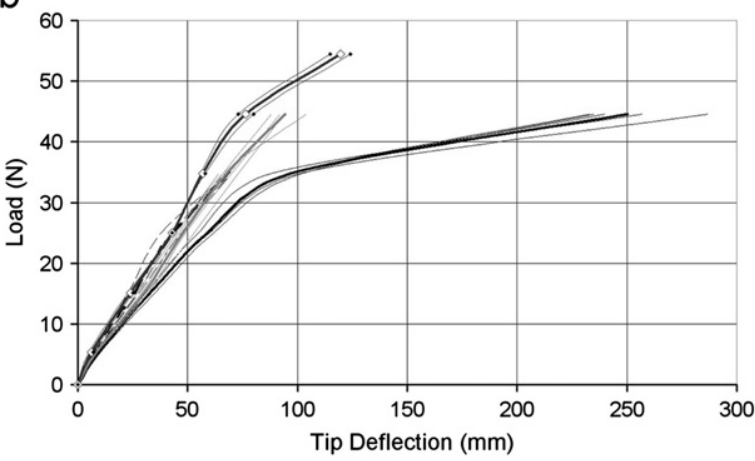

Fig. 16. Tip deflection displacement for $60 \mathrm{~cm}$ tube. 
inflatable tube's ability to resist buckling has increased as a result. Therefore the benefit of the structural stiffeners becomes less apparent.

The deflection data for the $0.4 \mathrm{~m}$ tube is shown in Fig. 15. The results for the lower pressure (Fig. 15(a)) displays a very clear repeat of the trends found in Fig. 14(a). The buckling point of each structural configuration is clearly represented along with the applicability of the average result. At the higher pressure the inflatable tube is more resistant to buckling but the stiffness relationship for the weaker two configurations is less than that displayed by the stiffer configurations. As a result, no clear buckling point can be identified for the inflatable tube configuration.

Fig. 16 shows the equivalent deflection data for the $0.6 \mathrm{~m}$ tube and displays similar data trends. However due to the increased length of the tube, the buckling loads of the inflatable tube configuration become more defined. A clear buckling point can now be identified for the stiffest configuration as displayed in Fig. 16(b).

\section{Performance comparison}

In order to analyse the data equivalently for all tube lengths the root bending moment can be determined for each case and plotted against the tip deflection. This allows the root bending moment magnitude that causes the buckling failure to be determined. Fig. 17 displays the average tip deflection data with respect to the applied root bending moment for the inflatable tube configuration.

It can be seen from this data that, as bending theory suggests, the bending moment to cause a buckling failure is independent of tube length and is more or less consistent for each inflation pressure. The exact magnitude that defines the onset of buckling is somewhat subjective (for this stage of testing), but it is around 8 and $19 \mathrm{~N} \mathrm{~m}$ for 10 and 15 PSI, respectively. These values can now be used to assess the performance benefits of using the tape spring structural stiffeners.

Fig. 18 shows these average inflatable tube tip deflection results along with the other configuration tip deflection averages at a pressure of 10 PSI.

By studying the small tape pair deflection data it can be seen that the onset of buckling occurs at a root bending

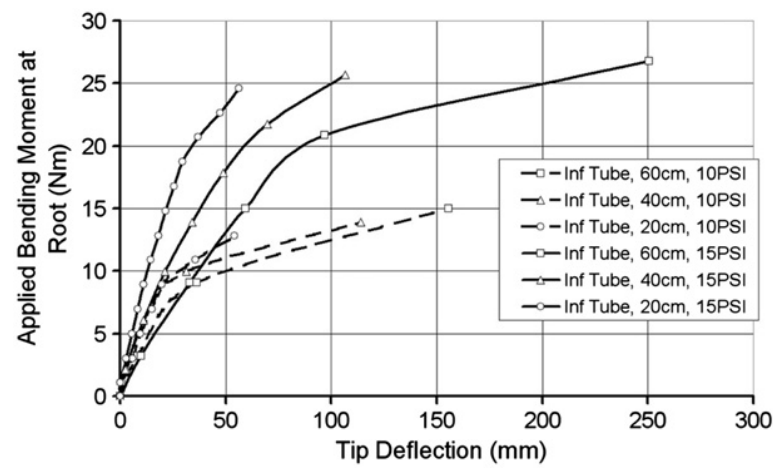

Fig. 17. Applied root bending moment against tip deflection for the inflatable tube configuration.

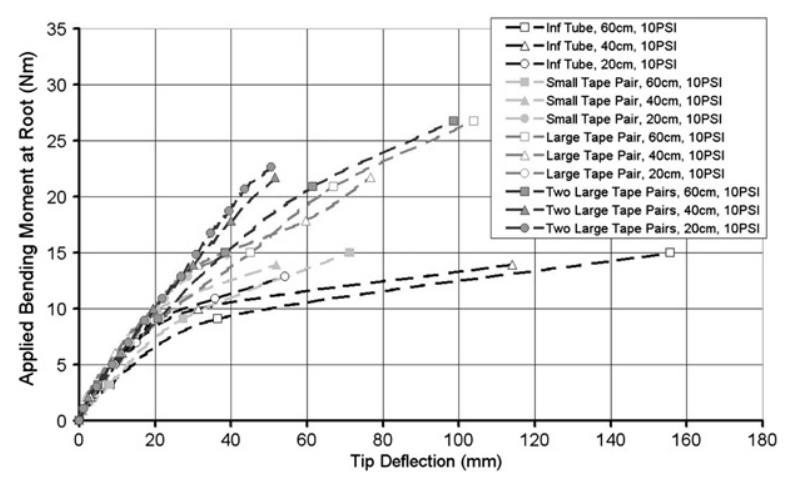

Fig. 18. Applied root bending moment against tip deflection for all configurations at 10 PSI.

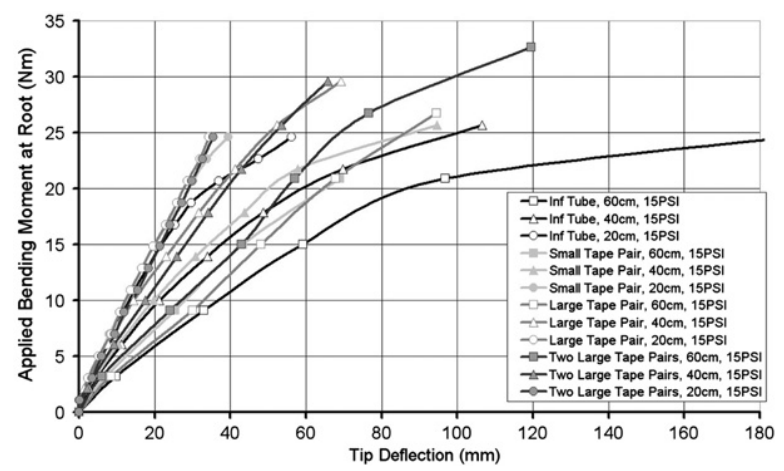

Fig. 19. Applied root bending moment against tip deflection for all configurations at 15 PSI.

moment magnitude of between 8 and $10 \mathrm{~N} \mathrm{~m}$. The $0.6 \mathrm{~m}$ displacement result for this configuration shows a relatively smooth curve, whereas the shorter lengths show a more defined transition between 9 and $10 \mathrm{~N} \mathrm{~m}$. A figure of $9 \mathrm{~N} \mathrm{~m}$ will therefore be used as a conservative estimate for this configuration. For the third configuration, the long tube length displacement result is not linear, and hence cannot be used with any certainty as to the point of buckling. However the shorter tube lengths both display a clear gradient change between 13 and $14 \mathrm{~N} \mathrm{~m}$, resulting in a conservative value of $13 \mathrm{~N} \mathrm{~m}$ for further analysis. The fourth configuration again displays a smoother gradient change for the long tube length, making a single buckling point hard to determine. The shorter lengths suggest a value of around $20 \mathrm{~N} \mathrm{~m}$.

The equivalent data for the higher inflation pressure is shown in Fig. 19.

As with the lower inflation pressure deflection data the longer tube lengths are inconclusive when attempting to determine the buckling root moment for the small and large tape pairs. However for the small tape pair the maximum bending moment applied to the long tape length was $21 \mathrm{~N} \mathrm{~m}$. This value is around the onset of buckling for this configuration as shown by the shorter tube lengths. The point of buckling for the stiffer two configurations at this higher pressure are harder to assess as the maximum tip force does not exceed the buckling moment for the 
Table 3

Root bending moment for failure comparision.

\begin{tabular}{lll}
\hline Configuration & $\begin{array}{l}\text { Root bending moment for buckling } \\
\text { failure }\left(\% \text { change with respect to }{ }^{*}\right)\end{array}$ \\
\cline { 2 - 3 } & 10 PSI & 15 PSI \\
\hline Inf tube & $8^{*}$ & $19(137.5)$ \\
Small tape pair & $9(12.5)$ & 21 \\
Large tape pair & $13(62.5)$ & 25 \\
Two large tape pairs & $20(150)$ & 25 \\
\hline
\end{tabular}

shorter lengths. However, using the longer lengths it can be concluded that the root bending moment for failure, for these two configurations is not less than $25 \mathrm{~N} \mathrm{~m}$. These root bending moment magnitudes that result in a buckling failure are summarised in Table 3.

By studying the mass data shown previously in Table 2 it can be seen that the mass of the inflatable tube without stiffeners can be modelled as a function of length using Eq. (1), where the mass and length is measured in grams and metres, respectively:

Mass $_{\text {tube }}=237.5 \times$ length +67.7

However, it should be reminded that the masses in Table 2 include the end plate mass of $46 \mathrm{~g}$. If we remove this from our mass estimation then the mass of the tube can be found using Eq. (2):

Mass $_{\text {tube }}=237.5 \times$ length +21.7

These functions enable the mass of the tube, which changes with length, and the mass of the end cap to be determined. If the tapes spring masses per unit length are known to be 10 and $21 \mathrm{~g} / \mathrm{m}$ for the small and large tapes, respectively, then these values can be ratioed with the length variable of the tube mass to determine a percentage mass increase which is independent of length. This results in the percentage mass increases shown in Table 4 .

These values can be compared with the root bending moment for failure values shown in Table 3 which are also independent of tube length.

From the data shown it is known that increasing the structural stiffening in the tube increases the tubes ability to resist buckling. However, we can use the data displayed in Table 3 to initially quantify this effect and to offset this benefit with the mass increase shown in Table 4. By adding small tape springs into the structure we have gained a strength increase of $12.5 \%$ at a mass increase of $8.4 \%$. As these numbers are only approximate figures we can state that for small stiffeners the strength and mass increases are almost comparable. As more stiffeners are applied to the structure, the system becomes more efficient, with the percentage stiffness increase rising faster than the mass percentage. However, the stiffness increase for the two large tape pair configurations is now comparable to an increase in internal pressure to 15 PSI. This was achieved with a mass increase of about $35 \%$. The use of tape spring
Table 4

Percentage mass increase of inflatable tube.

\begin{tabular}{lc}
\hline Configuration & \% mass increase of tube length \\
\hline Small tape pair & 8.4 \\
Large tape pair & 17.7 \\
Two large tape pairs & 35.4 \\
\hline
\end{tabular}

stiffeners therefore becomes a tradeoff between the mass increase of the tape spring stiffeners and the mass increase required to provide and sustain an equivalent higher pressure system.

\section{Conclusions and further work}

This paper has outlined the results of an initial test program at the University of Southampton to quantify the stiffness and mass tradeoff of inflatable hybrid structures. This experimental program incorporated a total of 120 separate loading tests on four different structural boom sections, allowing the buckling loads and respective masses to be determined, enabling an initial comparison of higher pressure systems and hybrid structures. It can be concluded that both approaches are effective at increasing the boom stiffness and the optimum structure is dependant on the mass required to achieve and sustain the higher pressure system. Further work is underway into the more detailed aspects of the configuration, such as more rigid tape spring root and tube attachments, requiring the construction of many specimens.

\section{References}

[1] S.E. Scarborough, D.P. Cadogan, Rigidizable materials for inflatable space and terrestrial structures, in: Proceedings of the 50th International SAMPE Symposium and Exhibition, May 1-5, Long Beach, CA, USA, 2005.

[2] H. de la Fuente, J.L. Raboin, G.R. Spexarth, G.D. Valle, TransHab: NASA's large-scale inflatable spacecraft, in: Proceedings of the 41st AIAA/ ASME/ASCE/AHS/ASC Structures, Structural Dynamics, and Materials Conference, April 3-6, Atlanta, GA, USA, 2000.

[3] M. Lou, H. Fang, L. Hsia, A combined analytical and experimental study on space inflatable booms, Proceedings of the IEEE Aerospace Conference 2 (2000) 503-512.

[4] J. Huang, The development of inflatable array antennas, IEEE Antennas and Propagation Magazine 43 (4) (2001) 44-50.

[5] S.J.I. Walker, G.S. Aglietti, Modeling the hinge moment of skew mounted tape spring folds, Journal of Aerospace Engineering 20 (2) (2007) 102-115.

[6] D. Givois, J. Sicre, T. Mazoyer, A low cost hinge for appendices deployment: design, test and applications, in; Proceedings of the Ninth European Space Mechanisms and Tribology Symposium ESA/ ESTEC, 2001

[7] D. Cadogan, T. Smith, R. Lee, S. Scarborough, D. Graziosi, Inflatable and rigidizable wing components for unmanned aerial vehicles, in: Proceedings of the 44th AIAA/ASME/ASCE/AHS/ASC Structures, Structural Dynamics, and Materials Conference, April 7-10, Norfolk, VA, United States, 2003, pp. 3688-3695.

[8] Fibermax Stabilized Nylon, Challenge Sailcloth Sales Brochure, 2009

[9] V. Firt, Statics, Formfinding and Dynamics of Air Supported Membrane Structures, Martinus Nijhoff Publishers, 1983. 